\title{
A importância do tratamento odontológico no pré-natal
}

\author{
The importance of prenatal dental treatment
}

Recebido: 21/10/2021 | Revisado: 25/10/2021 | Aceito: 29/10/2021 | Publicado: 31/10/2021

Camila de Oliveira Lyra

Universidade Brasil, Brasil

E-mail: camilaoliveiralyra.odonto@gmail.com

Roselaine Pessoa Alexandre

Universidade Brasil, Brasil

E-mail: roselainealexandre00@ gmail.com

Ana Lúcia de Freitas Sena

Universidade Brasil, Brasil

E-mail: analuciafreitas09@gmail.com

José Lucas Martins

Universidade Brasil, Brasil

E-mail: José.lucas@universidadebrasil.edu.br

\begin{abstract}
Resumo
O presente trabalho tem como objetivo principal identificar a percepção de gestantes sobre o tratamento odontológico, especialmente no que se refere a sua concepção de saúde bucal e conhecimento da relação entre sua saúde bucal e a gestação. A gravidez é uma fase importante na vida de qualquer mulher. As gestantes fazem parte do grupo de pacientes com necessidades especiais e merecem uma atenção especial, pois dentre tantas mudanças, ocorrem também alteraç̃oes odontológicas, a prevenção tem que ser prioridade e durante toda a gestação, a mulher deve ser orientada a manter normalmente a escovação e hábitos de higiene, sem hesitar em procurar o dentista surgindo problemas na cavidade bucal, estes problemas exercem influência para o feto e para a mãe, principalmente a doença periodontal e infecções. O tratamento deve ser instituído em qualquer momento da gestação. Entretanto, o período mais seguro e indicado para o tratamento eletivo é o segundo trimestre de gestação, mas em casos de urgência devem ser tratados independente do período gestacional, o cirurgião dentista está apto e deve estar preparado para atender urgências endodônticas, extrações ou drenagens, independente do estágio da gravidez. Ainda é perceptível que mulheres gestantes desconhecerem a importância do pré-natal odontológico, com medos ou mitos. O pré-natal odontológico tanto nas UBS quanto nas clínicas odontológicas particulares é de total responsabilidade dos profissionais levarem até essas pacientes gestantes essa informação, sendo assim uma boa oportunidade em promover saúde trazendo eficácia no tratamento e atendimento. Na metodologia foram consideradas publicações entre 2005 a 2019, com base em dados inglês e português: Google Acadêmico, PubMed, Scielo (Scientific Eletronic Library Online) e BDTD (Biblioteca digital brasileira de tese e dissertações).

Palavras-chave: Odontologia; Grávida; Saúde bucal; Pré-natal.
\end{abstract}

\begin{abstract}
The maisn objective of this study is to identify the perception of pregnant women about dental treatment, especially with regard to their conception of oral health and knowledge of the relationship between their oral health and pregnancy.Pregnancy is an important phase in any woman's life. The pregnant women are part of the group of patients with special needs and deserve special attention, while the attention to women, prevention has to be a priority and throughout pregnancy, hygiene habits, no problems in doing the dentist, in oral surgery, these problems exert influence on the child and the mother, mainly periodontal disease and infections. Development should be instituted at any time during gestation. However, the safest and most suitable period for elective treatment is the second trimester of gestation, but in cases of urgency, it must be independent of the gestational period, the dental surgeon is fit and must be prepared to attend endodontic emergencies, extractions or drainage, regardless of the stage of pregnancy. It is still perceptible that women manage the inequality of importance of the prenatal dentistry, with fears or myths. The prenatal dentistry in both UBS and dental clinics is a complete initiative of all professionals who receive patients with this information, thus being an opportunity to promote health bringing success without treatment and care. The methodologt considered publications between 2005 and 2019, based on English and Portuguese data: Academic Google, PubMed, Scielo (Scientific Eletronic Library Online) and BDTD (Brazilian Digital Thesis and Dissertation Library).
\end{abstract}

Keywords: Dentistry; Pregnant; Oral health; Prenatal. 


\section{Introdução}

O programa Brasil Sorridente foi criado em 2004, desde então ampliou o número de atendimentos odontológicos e com ele ações de promoção e prevenção de saúde bucal, trazendo mais eficácia no atendimento das UBS. Mas ainda é perceptível que muitas mulheres desconhecem a importância do pré-natal odontológico. Por isso é essencial o acolhimento e integração dos profissionais, conscientizando a paciente da importância do pré-natal odontológico, evitando assim complicações futuras. (BATISTELLA et al. 2006).

A gestante passa por um processo de alterações físicas, hormonais e psicológicas, necessitando de orientações sobre tais mudanças. Existem muitos mitos em relação ao atendimento odontológico de gestantes, tanto por parte dos cirurgiões dentistas, quanto por parte das futuras mães. (TRENTIN et al. 2007).

As gestantes merecem uma atenção especial, pois dentre tantas mudanças, ocorrem também alterações odontológicas, podendo ser elas gengivite associada ou não a má higiene bucal, acumulo de biofilme, tumores gestacionais, mobilidade dentária associada a doenças periodontais, xerostomia durante a gestação. (REIS et al. 2010).

A prevenção tem que ser prioridade, surgindo problemas na cavidade bucal, que tenham influência para o feto e para a mãe, principalmente se estiver contribuindo para infecções o tratamento deve ser instituído em qualquer momento da gestação (DA SILVEIRA et al. 2016). O período mais seguro e indicado para o tratamento é a partir do segundo mês de gestação, mas em casos de urgência devem ser tratados independente do período gestacional. (FAGUNDES et al. 2016).

Amparar a gestante no início da gestação é o principal objetivo do pré-natal odontológico, oferecendo atenção total a saúde mãe e filho. A finalidade da assistência odontológica é conservar e restaurar a saúde bucal, através da prevenção e promoção da saúde. Ainda temos pouca relação entre profissionais, médicos, enfermeiros e dentistas, para conscientização da importância do pré-natal odontológico. (MONTEIRO et al 2018)

Podemos constatar, que na prática, ainda com políticas de saúde bucal ativos, são baixos os índices de atendimento pré-natal odontológico integral como sugere a promoção de saúde. (ZEMOLIN et al. 2017).

O presente trabalho tem como objetivo principal identificar a percepção de gestantes sobre o tratamento odontológico, especialmente no que se refere a sua concepção de saúde bucal e conhecimento da relação entre sua saúde bucal e a gestação.

\section{Metodologia}

Este estudo utiliza como natureza qualitativa, visando compreender como a equipe multiprofissional ligada à gestante, pode influenciar na oferta, procura e adesão a esse cuidado; bem como o grau de conhecimento de gestantes quanto à prevenção, consequências e oportunidade de tratamento. (PEREIRA, et al. 2018).

Os critérios de inclusão foram os seguintes: artigos e revistas, disponível nos idiomas em língua inglesa e portuguesa nos sites como: Google Acadêmico, PubMed, Scielo (Scientific Eletronic Library Online) e BDTD (Biblioteca digital brasileira de tese e dissertações), contextos disponíveis em meios eletrônicos, que foram publicados entre 2005 a 2019 . Os critérios de exclusão: relatórios e publicações sem fontes confiáveis, materiais com disponibilidade incompleta que não abordava com clareza o tema.

\section{Revisão de Literatura}

A gravidez é um evento fisiológico com alterações orgânicas naturais, o que obriga os profissionais de saúde a obterem conhecimentos específicos sobre o cuidado humanizado e integral. É mais fácil aceitar novos conhecimentos 
relacionados a sua saúde e a saúde infantil, portanto, é importante prestar serviços na perspectiva da promoção e educação em saúde. (

Batistella et al., em 2006, realizaram uma entrevista com gestantes, tendo em vista as alterações que ocorrem no organismo da mulher durante a gestação e a importância da Odontologia preventiva. Com isto foi realizado questionários a 200 gestantes sendo 1000 questionários em gestantes que realizaram atendimento na UBS e 100 questionários aplicados na recepção de clínicas ginecológicas particulares. Como resultados observou-se que $75 \%$ das gestantes do SUS responderam que achavam bom e $85 \%$ das gestantes da clínica privada também consideram bom. Quantos as orientações recebidas sobre saúde bucal a maioria das entrevistadas não teve acesso a essas informações. A respeito de já terem ouvido falar em pré-natal odontológico, a maioria das gestantes $(86,6 \%)$ entrevistadas não ouviu falar do assunto e não houve diferença estatisticamente significante entre os dois grupos estudados. Embora a maioria considere como bom o tratamento, ainda existe muito medo da anestesia, de prejudicar o bebê e de hemorragias (37\% das respostas do SUS e 19\% das respostas das clínicas particulares). Para nossa surpresa 3,1\% das respostas das gestantes do SUS relataram ter recebido informações do próprio dentista de que não deveriam realizar tratamento odontológico neste período, mostrando a desinformação também dentro da classe odontológica. Os resultados permitem concluir que o conhecimento das gestantes sobre os cuidados odontológicos na fase da gestação, na cidade, é baixo. (BATISTELLA et al. 2006).

Trentin et. al em 2007, investigaram a correlação entre doença periodontal em gestantes, fatores de risco para essa doença e a ocorrência do parto prematuro. Para isto participaram da pesquisa 143 mulheres com idade acima de 18 anos, das quais 70 tiveram parto prematuro (menos de 37 semanas de gestação) e 73, parto a termo (37 semanas ou mais de gestação). Foram utilizados instrumentos de avaliação clínica e um questionário sociodemográfico para a identificação de variáveis como idade, escolaridade, nível socioeconômico e hábitos de higiene bucal. O exame clínico foi realizado por dois examinadores treinados, utilizando o índice Periodontal Screening and Recording (PSR). Como resultado foi possível verificar que das 107 participantes encontram-se na faixa etária de 18 a 30 anos. Em relação a faixa etária de 18 a 30 anos, 14 participantes do grupo apresentam saúde periodontal, no grupo etário acima de 40 anos nenhuma participante apresenta saúde periodontal. Pelos dados analisados, concluiu-se que o tratamento periodontal prévio a faixa etária de 31 a 40 anos influenciariam de maneira positiva nos resultados do grupo, caso visto que as participantes acima de 31 anos e a que não haviam recebido tratamento periodontal prévio apresentam maior risco de terem parto prematuro.

Reis et al. em 2010, realizaram uma revisão de literatura sobre a importância da educação da saúde bucal no período gestacional. Para isto considera-se importante o programa de atenção à saúde da mulher, conforme recomendações atuais diretrizes da política nacional saúde bucal. Destaca-se a promoção da saúde bucal na gestante a educação em saúde bucal. A abordagem atual das ações odontológica fundamentada na promoção de saúde bucal, busca o tratamento integral vida individuação baseado principalmente em ações educativas preventiva. Aos autores concluíram que a partir do trabalho de educação em saúde desenvolvida pelos profissionais de saúde no pré-natal, a mulher poderá como agente multiplicador de informações preventivas de promoção de saúde bucal, informações relevantes sobre a atenção a saúde em gestantes apontam a educação em saúde como estratégia da saúde bucal.

Segundo Silveira, Abraham e Fernandes em 2016 realizaram um estudo com o objetivo de identificar a percepção das gestantes que não aderem ao tratamento odontológico, a respeito do mesmo, especialmente no que se refere a sua concepção de saúde bucal e conhecimento da relação entre saúde bucal, para tanto, realizou-se uma pesquisa qualitativa onde os dados das entrevistas foram gravados em áudio, transcritos e analisados, foram entrevistadas oito gestantes de duas unidades de Estratégia de Saúde da Família que realizavam pré-natal com histórico de não adesão ao tratamento odontológico. A média de idade das gestantes foi de vinte e três anos com mínimo de dezenove e máximo de trinta e cinco anos. Foram identificadas as 
seguintes categorias de análise: a) Medo de dentista; b) Necessidade de assistência; b) Desinformação; c) Dificuldade de acesso e d) Falta de adesão ao tratamento odontológico. Conclui-se que a baixa aderência ao cuidado odontológico entre as gestantes participantes deste estudo se associa ao medo de sentir dor ou receio do tratamento afetar seu bebê. As gestantes compreendem o tratamento odontológico limitado a procedimentos cirúrgico-restauradores.

Fagundes, Oliveira e Santos em 2015, O baixo peso ao nascer se deve a múltiplos fatores, que são responsáveis pelos níveis de mortalidade e morbidade infantil, sendo amplamente estudados, inclusive na odontologia, a fim de reduzir seus danos. Falta de assistência odontológica ao nível curativo, índice de massa corporal materno menor que $20 \mathrm{~kg}$ aumentam as chances da ocorrência do baixo peso ao nascer. Ausência da assistência odontológica no pré-natal não é capaz de favorecer isoladamente o baixo peso ao nascer porque os fatores associados são muitos. Por isso Fagundes (2015), defende que novos estudos devem ser realizados para fundamentar políticas públicas direcionadas a saúde materno-infantil. Assistência odontológica no pré-natal e o baixo peso ao nascer.

Monteiro et al. em 2012, a fase gestacional é um período em que ocorrem diversas alterações físicas, hormonais e psicológicas, e, o exame dos hábitos de higiene oral dessas gestantes são capazes de prevenir problemas orais como a periodontite, visto que, a orientação educativa-preventiva promove condições de saúde as gestantes, evitando risco ao feto, como o parto prematuro e o baixo peso ao nascimento. Questionado se as gestantes se as gestantes receberam informações sobre as possíveis alterações bucais que ocorrem durante o período gestacional e destas 47 mulheres (62\%) não receberam nenhuma informação e apenas 28 (37,3\%) foram informados, é de grande importância que o cirurgião dentista tenha conhecimento sobre as alterações bucais e sistêmicas deste paciente, e que a gestante busque o atendimento e se tenha consciência de que esses problemas podem ser evitados.

Zemolin et al. em 2017, durante o período pré-natal odontológico algumas informações são perdidas no que tange aos cuidados da higiene oral da criança e a relevância da amamentação para o desenvolvimento das estruturas do sistema estomatognático, uso de mamadeira e chupetas e o risco de transmissão de bactérias para o bebê através da saliva dos membros familiares devem ser passadas. Das entrevistadas, 66\% haviam sido orientandas quanto a saúde bucal, tendo a mesma porcentagem procurado atendimento odontológico. Destas $57 \%$ sentiram um pouco de desconforto com o posicionamento da cadeira enquanto $43 \%$ não sentiram nenhum desconforto. No entanto, relata-se que é importante o papel do dentista sobre o cuidado da gestante e o feto tanto no serviço público quanto no particular sobre a higiene bucal em seu período gestacional.

Santana et al. em 2018, no período gestacional ocorrem alterações hormonais, a doença periodontal eleva 4,8 vezes o risco do parto prematuro e bebê de baixo peso. Os profissionais da odontologia têm que ter o conhecimento para um atendimento diferenciado e efetivo. Da mesma maneira que o acompanhamento com o obstetra é indispensável, visitas regulares ao dentista também são de suma importância. Isso acontece porque a saúde bucal da mãe influência diretamente na saúde do bebê. Problemas periodontais podem levar a um parto prematuro e o nascimento da criança abaixo do peso. Nessa perspectiva, relata-se que a criação do elo dentista-gestante, é primordial, visto que num segundo momento poderá ser substituído, pelo vínculo dentista- mãe- bebê e futuramente dentista - criança, dentista- adulto, conformando um ciclo de saúde que recomeça a cada nova geração, estendendo seus benefícios a todos. (BRASIL 2012).

Codato e Melchior em 2008, De acordo com a entrevista com feitas com mulheres gestantes que usam do sistema único de saúde e clinicas privadas mostram que ainda muitas gestantes temem ao tratamento odontológico. Sugere-se com este dado que a dificuldade do acesso da população de conseguir tratamento odontológico aponta a necessidade de planejamento e reavaliação da atenção odontológica prestada, bem como a necessidade de ampliação do número de equipes existentes para melhorar a oferta e, por conseguinte, o acesso da população aos serviços. Entre os dois grupos entrevistados, existem mitos e 
restrições fortemente arraigados sobre atendimento odontológico clínico durante a gravidez, relacionados a preocupação com a possibilidade de sequelas, principalmente, no que tange a saúde do bebê nos primeiros meses de vida.

Figueira et al. em 2010 descreve que no período gestacional são muito comuns os hábitos alimentares inadequados e a precária higiene bucal, o que as torna fatores de risco para o surgimento da cárie dentária e doença periodontal. O objetivo deste trabalho foi identificar as principais crenças das gestantes que tem influência na procura pelo atendimento odontológico e na admissão de cuidados relativos à saúde bucal. Para tanto, utilizou-se o referencial teórico o Modelo de Crenças em Saúde (MCS) numa perspectiva qualitativa. Foram entrevistadas 20 gestantes usuárias em um Centro de Saúde. Como resultados foram observados a relação entre as dimensões do MCS e o comportamento da população estudada em relação a saúde bucal. Conclui-se que o MCS é um instrumento útil para compreender o comportamento em saúde bucal e para planejar ações educativas. (BATISTELLA et al. 2006).

Nascimento et al. em 2012, descreveu-se de forma a avaliativa à respeito da conduta de gestantes que foram atendidas nos consultórios médicos que se localizavam em Alfenas (MG) sobre suas percepções ao tratamento odontológico. Foi feito um estudo transversal e quantitativo com 100 gestantes que procuraram atendimento médico em consultórios particulares e do SUS de deste município. Através da aplicação de questionários contendo 18 questões cada, foram realizadas as entrevistas. Observou-se que a maioria das gestantes realizavam três escovações/ dia em média e utilizam o fio dental na mesma proporção Com os resultados, viu-se que vinte e dois por cento das entrevistadas acusou sensibilidade à escovação, trinta e cinco por cento, sangramento fácil e vinte e quatro por cento apresentou gengiva edemaciada e medo da exposição aos raios X. Concluiu- se que as gestantes apresentam bons hábitos de higiene bucal, contudo sentem medos e desconfortos relacionados a procedimentos odontológicos. Algumas alterações sistêmicas que ocorrem, influenciando a atividade bucal que se devem em fato a gravidez, além das náuseas e vômitos comuns deste ciclo, podem ser vistas: hipersalivação, ganho de peso, aumento da urina, função respiratória funciona de forma restringida, hipoglicemia, diminuição ou aumento dos batimentos cardíacos e até mesmo síncopes.

Soares et al em 2009, A fim de discutir à respeito da inclusão de gestantes com gravidez de alto risco no pré-natal odontológico confeccionou-se este trabalho enfatizando-se a ideia de uma equipe multidisciplinar como coadjuvante a fim de desvencilhar a população de mitos populares perante o atendimento nas classes médica e odontológica. Para compor este trabalho foi realizada uma revisão de literatura utilizando para tanto, o levantamento de 48 artigos datados de 1981 a 2008 , utilizando as bases acadêmicas de busca para pesquisa, foram usadas as palavras chave: Gestação. Odontologia. Pré-natal. Inclusão. Ensino de graduação. Como resultado, evidencia-se a necessidade de investimentos em educação direcionados aos profissionais de saúde, de graduação e pós-graduação, visto que o conhecimento e a atualização sobre a prática em questão contribuiriam com o melhor atendimento frente a essa parcela de gestantes, que sendo de alto risco ou não, não deixam de ser pacientes com necessidades especiais e requerem uma atenção odontológica minuciosa. A odontologia atual lança mão de recursos tecnológicos que permitem ao cirurgião dentista tratar a gestante sem problemas. Conclusão: A interação entre a área medica e odontológica se faz necessária para que a gestante seja percebida como um todo. Essa interação multidisciplinar beneficiaria dentistas e médicos, e principalmente as pacientes gestantes.

Oliveira et al. em 2014, durante a gestação é extremamente necessário fazer a manutenção da saúde oral. Porém, este é um tema pouco difundido nas políticas públicas de atenção à saúde das gestantes brasileiras. Uma parcela considerável da população não possui acesso a informações ou sabe quais são as alterações bucais características deste período. Os estudos científicos apontam que a atenção odontológica durante a gestação se é altamente indicada visto que muitas mulheres apresentam perturbações orais decorrentes das alterações hormonais. Existem fortes evidências da associação entre a doença periodontal e a prematuridade e o baixo peso do bebê ao nascer. 
Trevisan e Pinto em 2013, Durante a análise dos questionários preenchidos pelas gestantes, percebeu-se a adesão aos cuidados caseiros são mais adotadas em relação ao cuidado profissional, e isto é influenciado por múltiplos fatores como: auto eficácia, facilidade para realização e susceptibilidade, também demonstrou-se que a escovação dentária foi a medida unicamente realizada com mais frequência, no que tange a busca por atendimento odontológico, a busca por tratamento é muito mais resolutivas do que preventiva, a procura é pela remoção da dor e manutenção ou reestabelecimento da estética. Entretanto, o período gestacional não mudou a percepção das gestantes sobre o processo saúde-doença em relação a cuidados preventivos e benefícios em relação ao pré-natal no que tange a odontologia, exceto em relação à percepção de maiores barreiras para se concretizar o tratamento. Desta maneira, salienta-se que o trabalho do cirurgião dentista é de fundamental importância para demonstrar a importância do tratamento odontológico, a fim de evitar hábitos inadequados e doenças bucais indesejáveis durante este período. No mais, enfocando a necessidade do acompanhamento odontológico nessa fase, faz parte de um trabalho não só curativo, mas preventivo a buscando ferramentas para que propiciem à gestante qualidade de vida. Para tanto, deve haver uma íntima parceria entre ginecologistas, pediatras e dentistas, o que é de suma importância, tendo em vista que o receio dos dentistas em realizar tratamentos em gestantes e o total desconhecimento médico sobre os procedimentos odontológicos na paciente gestante. Então, cabe a estas áreas da saúde zelar para assegurar a gestante receba tratamento integral e multidisciplinar a saúde, assim contribuindo para previnir muitos problemas bucais que acometem sistemicamente a saúde da criança, visto que as crianças usualmente visitam o médico antes da odontopediatria

\section{Discussão}

Percebeu-se nesta pesquisa que dos 20 artigos pesquisados, todos comentam as alterações fisiológicas durante a gestação. Alguns autores comentam que estas transformações vão acontecer em todo o organismo, inclusive sobre a cavidade bucal e na fisiologia oral. Pesquisas mostram que $60 \%$ a $70 \%$ das gestantes podem ter gengivites, tumores gestacionais, mobilidade dentária, xerostomia ou pode ocorrer aumento de salivação. Dentre os 20 artigos pesquisados, no que tange a educação durante o período gestacional, seis autores defendem a necessidade de se trabalhar com educação em saúde com estas mulheres por toda a equipe no pré-natal. (TRENTIN et al. 2007, REIS et al. 2010, FAGUNDES et al. 2015).

De acordo com Trevisan e mais cinco autores, a importância do zelo com a saúde bucal durante a gravidez é desconhecido pelas gestantes, o que evidencia a necessidade de se trabalhar com educação em saúde com estas mulheres por toda a equipe no pré-natal. Quando se relata sobre a incidência da doença periodontal nas gestantes, dos 20 artigos pesquisados, apenas três artigos acreditam que desordens periodontais afetam as mulheres no período de gestação devido a ação hormonal e a falta de acompanhamento.

Segundo Santana e mais dois autores, a gestante com doença periodontal tem 4,8 vezes o risco de parto prematuro e baixo peso infantil, o que trará prejuízos na mineralização dos dentes. Ao citar o conhecimento das gestantes em relação a importância dos cuidados odontológicos no pré natal, dos 20 autores, três autores ressaltam o papel do dentista sobre o cuidado da gestante e o feto tanto no serviço público quanto no particular sobre a higiene bucal em seu período gestacional. (BATISTELLA et al. 2006, ZEMOLIN et al. 2017).

Segundo Batistella e mais dois autores, o conhecimento das gestantes sobre os cuidados odontológicos na fase da gestação é baixo. No tocante a insegurança das gestantes, dentre os autores, três deles acreditam que as gestantes apresentam bons hábitos de higiene bucal, se sentem desconfortáveis em relação a procedimentos odontológicos. (SILVEIRA et al. 2016, CODATO et al. 20082, NASCIMENTO et al. 2012).

Segundo Silveira a baixa aderência ao cuidado odontológico entre as gestantes participantes deste estudo se associa ao medo de sentir dor ou receio do tratamento afetar seu bebê. Quando se cita os mitos do cuidado odontológico durante a 
gestação, os autores, acreditam que devido a fatores de origem psicológica por sentirem medo relacionado a procedimentos odontológicos. De acordo com Codato e mais dois autores, existem mitos e restrições fortemente arraigados sobre atendimento odontológico clínico durante a gravidez, relacionados a preocupação com a possibilidade de sequelas a saúde do bebê. (NASCIMENTO et al. 2012, SOARES et al. 2009).

\section{Considerações Finais}

Com este trabalho é possível concluir que, durante o período gravídico, a propensão ao aparecimento de alterações bucais, como: hipersalivação, problemas periodontais, cáries e tumor gravídico e abcessos, que geram quadros infecciosos podendo levar ao parto prematuro além do baixo peso da criança.

Ainda que o nesta fase as gestantes estejam mais abertas a sugestões e tratamentos de saúde, o acesso das gestantes ao sistema de saúde durante o período de pré natal, seja ele público ou particular ainda é envolto em vários mitos, onde se teme que os cuidados odontológicos possam prejudicar o desenvolvimento da criança no período gestacional, o que faz com que a procura deste serviço se dê somente em casos de urgência odontológica; antes disso as mães preferem cuidados paliativos em casa, se valendo da escovação como método preventivo.

Isto posta, ao incluírem-se nos programas de saúde bucal, ações voltadas à saúde mulher durante o período da gravidez é de suma importância para que se promova a saúde integral de mãe e filho, visto que os benefícios se estenderão a mãe e ao filho, permitindo que ele tenha um desenvolvimento normal durante a gestação. Dessa forma, concluímos que, persiste a necessidade de orientações frequentes sobre cuidados com saúde bucal às gestantes, visto que estes não fazem parte da rotina na atenção pré-natal. Evidenciamos a necessidade do trabalho integrado com toda a equipe médica e odontológica para melhor esclarecimento sobre a relevância do tratamento odontológico.

\section{Referências}

BRASIL. Atenção ao pré-natal de baixo risco. Brasília (DF): Ministério da Saúde; 2012. (Cadernos de Atenção Básica n. 32).

BATISTELLA, F.I.D, IMPARATO, J.C.P, RAGGIO, D.P, \& CARVALHO A.S. Conhecimento das gestantes sobre saúde bucal; na rede pública e em consultórios particulares. Rev Gaucha Odontol, 2006; 54:67-73.

CODATO, L.A.B., NAKAMA, L, MELCHIOR, R. Percepções de gestantes sobre atenção odontológica durante a gravidez.Ciênc Saúde Coletiva. 2008; 13: $1075-1080$.

CODATA, L.A.B., NAKAMA, L, CORDONI JÚNIOR, L, \& HIGASI, M.S. Atenção odontológica à gestante: papel dos profissionais de saúde. Ciênc Saúde Colet [serial on the internet]. 2011 [cited 2019 Dec 16];16(4):2297- 301. http://www.scielo.br/pdf/csc/v16n4/v16n4a29.pdf

FAGUNDES, D.Q; OLIVEIRA, A.E. DOS S. N., \& EDSON, T. Assistência odontológica no pré-natal e o baixo peso ao nascer. Revista Brasileira de Pesquisa em Saúde/Brazilian Journal of Health Research, 2015.

FIGUEIRA T.R, FERREIRA E, SCHAAL V, \& MODENA C. O modelo de crenças em saúde e o processo saúde doença cuidado bucal por gestantes - Rev. Odontol. Bras. Central (2013) 22 (63).

MONTEIRO R.M, SCHERMAAP, AQUINO D.R, OLIVEIRA R.V, \& MARIOTTO A.R. Avaliação dos hábitos de higiene bucal de gestantes por trimestre de gestação. R Periodontia 2012;22(4):90-9.

NASCIMENTO P. E, DUARTE A. M, \& SOUZA F. Gestantes frente ao tratamento odontológico -Rev. Bras. Odontol., Rio de Janeiro, (2012) 6(9): 125-30

NASCIMENTO E.P, ANDRADE F.S, COSTAS A.M.D.D, \& TERRA F.S. Gestantes frente ao tratamento odontológico. Rev Bras Odontol 2012. 69(1):12530. Available from: http:// revodonto.bvsalud.org/pdf/rbo/v69n1/a28v69n1.pdf

OLIVEIRA, E. C. Atendimento odontológico a gestantes: a importância do conhecimento da saúde bucal. Revista de Iniciação Científica da Universidade Vale do Rio Verde, Três Corações, v.4, n.1, p. 11-23, 2014.

PEREIRA A. S. (2018). Metodologia da pesquisa científica.

REIS, D.M. Educação em saúde como estratégia de promoção de saúde bucal em gestantes. Ciência \& Saúde Coletiva. ABRASCO - Associação Brasileira de Saúde Coletiva, v. 15, n. 1, p. 269-276, 2010. <http://hdl.handle.net/11449/30253>. 
REIS F.G, \& ALMEIDA JRS. Percepção das gestantes sobre o pré-natal odontológico. Ciênc Saúde Colet. 2013;4(1):56-62.

SANTOS, C.G., \& PEREIRA, D.P.C. A Importância da Odontologia no Cuidado da Gestante: Revisão de Literatura. Id on Line Rev.Mult. Psic., Maio/2020, vol.14, n.50, p. 1212-1230. ISSN: 1981-1179.

SILVA, C. C. da; MARONEZE, M. C.; ZAMBERLAN, C.; \& SANTOS, B. Z. Capacitação sobre o pré-natal odontológico para profissionais da equipe de saúde: relato de experiência. Research, Society and Development, v. 9, n. 8, p. e204984481, $2020 . \quad$ DOI: 10.33448/rsd-v9i8.4481. em: https://rsdjournal.org/index.php/rsd/article/view/4481.

SILVEIRA J.L.G.C, ABRAHAN M.W, \& FERNANDES C.H. Gestação e saúde bucal: significado do cuidado em saúde bucal por gestantes não aderentes ao tratamento. Rev APS. 2016; 19(4):568-74. https://pesquisa.bvsalud.org/portal/resource/pt/biblio-832216.

SOARES - Pré natal odontológico: a inclusão do cirurgião dentista nas equipes de pré natal - Revista Interdisciplinar de Estudos Experimentais, (2009) 2(2): 53-57,

TRENTIN, M.S. Doença periodontal em gestantes e fatores de risco para o parto prematuro. Revista da Faculdade de Odontologia-UPF, v. 12 , n. 1, 2007.

TREVISAN C.L, \& PINTO A.A.M. Fatores que interferem no acesso e na adesão das gestantes ao tratamento odontológico. Arch Health Invest. 2013; 2(2):29-35. DOI: 10.21270/archi.v2i2.136

ZEMOLIN A.B, SASSI E.T, WILLMS G.P, RECH J.P, \& DALSOCHIO L. Gestantes: da saúde bucal a saúde geral. Revista Saúde Integrada, v. 10, n. 20, 2017.

KLOETEZEL M.K, HUEBNER C.E, \& MILOROM P. Referrals for dental care during pregnancy. J Midwifery Womens Health [serial on the internet]. 2011;56(2):110-7. https://www.ncbi. nlm.nih.gov/pmc/articles/PMC3074205/ 